\title{
AOR
}

Selected Papers of \#AoIR2020:

The 22nd Annual Conference of the

Association of Internet Researchers

Virtual Event / 13-16 Oct 2021

\section{STITCHING THE CURVE: PANDEMIC CRAFT AND FEMINIST DATA VISUALIZATION}

\author{
Abigail Moreshead \\ University of Central Florida \\ Lauren Rouse \\ University of Central Florida \\ Anastasia Salter \\ University of Central Florida

\section{Introduction}

Feminist scholars are increasingly drawing attention to the ways "big data" and data representations reinscribe gender and racial inequality, an issue made even more pressing by the role data has taken in our daily lives since the start of the COVID-19 pandemic (D'Ignazio and Klein 2020). And the myriad ways in which the pandemic has disproportionately impacted women makes these critiques even more crucial, particularly as this has occurred alongside widespread denial and misinformation. In this social media landscape, work such as the "Stitching the Curve" project, a textile pandemic visualization, offers an intersection between digital activism and craftivism, enabling a material, feminist response to an erasure and minimization of collective loss.

\section{Method}

To understand Stitching the Curve as a feminist intervention into data visualization, we examine the media coverage around the project, which includes the online blogs of the project's participants. Using critical technocultural discourse analysis (CTDA) as a guiding methodology, we consider simultaneously the feminist, activist framing and the influence of material and digital platforms on the cultural influence of the work (Brock 2018). This methodology draws our attention to the role of textile as technology, and the feminist efforts to reclaim a marginalized materiality's activist potential. Given the project's emphasis on drawing attention to marginalized data from mainstream periodicals, we focus on news articles and blog posts, which were coded for mentions of collaboration, data visualization, affective experience, activism, and materiality. Our goal 
is to understand the relationship between craftivism as data visualization and its relationship to a feminist ethics of data representation, such as those embraced in the FemTechNet Manifesto ("Manifesto - FemTechNet” 2021)

\section{Craft as Feminist Data Visualization}

In the introduction to an online exhibition entitled "Knitting Data," Michelson reminds viewers that "crafts are always communicating something," even if that communication is frequently trivialized along gendered labor binaries (Michelson 2020). Craftivism as data visualization exists at the intersection of feminist reclaiming of forms of labor and craft traditionally coded as feminine and feminist critiques of white cis-gender male dominated data science. Our project looks at how, specifically, COVID-19 data visualization craftivism operates at this intersection, building on a tradition of data visualization through craftivism being used to challenge the inherent biases of big data. Examples of this include the Tempestry Project, which uses yarn colors and knitting to represent climate data and Rebecca Michaelson's (USC) “Knitting Data: Data Visualization and Craft" project (Michelson 2020).

Pandemic visualization follows in the tradition of the AIDS Memorial Quilt, but given the scale and communities of impact, many of the visualizations here are depersonalized by comparison. In the specific project we are examining here, the University of Alberta library staff is representing the curve of COVID cases in Canada through a group knitting project that each member began working on from their own remote location once the lockdown began. They use colors to represent numerical data, with lighter colors corresponding to lower case numbers and vice versa. Each participant works on a section which represents the data of a specific Canadian province. The goal is to join each section together, eventually, to form a blanket and display it in a museum.

\section{Stitching the Curve as Craftivism}

The power of Stitching the Curve as a form of COVID craftivism is in its pairing of a traditionally feminized form of tactile labor with data visualization; specifically, data visualization in an era where "fake news," denial of COVID's reality, and undermining of public health experts has shifted the dialogue from how to best deal with the pandemic itself to how to debate about the very legitimacy of responding to it. Donald Trump's toxic masculinity was on full display throughout the initial COVID response, from his refusal to consistently wear a mask (reinforced by rhetoric on the right that has associated mask wearing with being unmanly) to his refusal to recognize any cost of the pandemic outside of the economic ones. Therefore, to visualize the "curve" is itself a feminist and social activist stance, by legitimizing the illness's toll through numerical data using a traditionally feminist medium.

The medium of knitting, an embodied form of data visualization, resonates with Gollihue and Xiong-Gum's assertion that textiles "hold special meaning in our lives as data centers, but also because of the ways they prompt interaction and reciprocity" (2020), while also resisting the illusion of objective neutrality that more typical forms of data visualization often portray (D'Ignazio and Klein 2020). Pearce notes that the library staff "share a great passion for information and we are always looking for new ways we can 
engage with it and make it more approachable for ourselves and our community" (Pearce 2020). Thus, knitting the curve is a creative act, an informative act, and an act of resistance.

\section{Making the Invisible Visible}

Brock argues that "CTDA has the additional task of operationalizing the computational object as discourse" (Brock 2018). Using the methodology of CTDA invites critique of both the message of Stitching the Curve-the visualization of pandemic data-but also the technologies that act as platforms for that data, which includes the scarf itself and the writing focused on the project. In looking at the role of blogging in communicating about the project, for instance, we see Stitching the Curve further removing data visualization from a rhetoric of distance and neutrality and toward a rhetoric of care and affect, as blogging is a space often associated with affective labor (Cummings 2019).

But other aspects of Stitching the Curve also resist the systemic patriarchy embedded in "big data" and embraces a collaborative feminist ethic. This is through the open sharing of their resources. Stitching the Curve's participants use Github to share the code through which they pull COVID-19 data and Google docs to share their crafting process, encouraging and enabling others to start their own groups to stitch the curve.

\section{Conclusion}

Blogging and knitting are frequently associated with craft and writing as an expression of the domestic and personal, relegated to a feminine and, consequently, minimized space of care and labor. Stitching the Curve makes a powerful statement in representing not only the oft-dismissed human cost of the COVID-19 pandemic, but also uses mediums of representation that challenge patriarchal "big data" collection and representational practices. Stitching the Curve makes data visualization a rhetoric of care.

\section{References}

Brock, André. 2018. "Critical Technocultural Discourse Analysis." New Media \& Society 20 (3): 1012-30. https://doi.org/10.1177/1461444816677532.

Cummings, Kelsey. 2019. “'But We Still Try': Affective Labor in the Corporate Mommy Blog." Feminist Media Studies 19 (1): 38-52. https://doi.org/10.1080/14680777.2017.1382548.

D’lgnazio, Catherine, and Lauren F. Klein. 2020. Data Feminism. MIT Press.

Gollihue, Krystin and Mai Nou Xiong-Gum. 2020. "Dataweaving: Textiles as Data Materialization." Kairos: A Journal of Rhetoric, Technology, and Pedagogy 25, no. 1. http://kairos.technorhetoric.net/25.1/disputatio/gollihue-xiong-gum/index.html

“Manifesto - FemTechNet." n.d. Accessed April 9, 2021. https://femtechnet.org/publications/manifesto/. 
Michelson, Rebecca. 2020. "Knitting Data: Craft as Recorded Information." Knitting Data: Data Visualization and Crafts. May 2020.

https://scalar.usc.edu/works/knitting-data/craft-as-recorded-information.

Pearce, Hanne. 2020. "Stitching the Curve: A UAlberta Library Data-Driven Making Project - News.Library.Ualberta.Ca." Accessed November 18, 2020. https://news.library.ualberta.ca/blog/2020/04/23/stitching-the-curve-a-ualbertalibrary-data-driven-making-project/. 